Grand Valley State University

ScholarWorks@GVSU

$10-2010$

\title{
Photodissociation of ozone in the Hartley band: Product state and angular distributions
}

\author{
George C. McBane \\ Grand Valley State University, mcbaneg@gvsu.edu \\ Luan T. Nguyen \\ Grand Valley State University \\ Reinhard Schinke \\ Max-Planck-Institut für Dynamik und Selbstorganisation, rschink@gwdg.de
}

Follow this and additional works at: https://scholarworks.gvsu.edu/chm_articles

Part of the Chemistry Commons

\section{ScholarWorks Citation}

McBane, George C.; Nguyen, Luan T.; and Schinke, Reinhard, "Photodissociation of ozone in the Hartley band: Product state and angular distributions" (2010). Peer Reviewed Articles. 34.

https://scholarworks.gvsu.edu/chm_articles/34

This Article is brought to you for free and open access by the Chemistry Department at ScholarWorks@GVSU. It has been accepted for inclusion in Peer Reviewed Articles by an authorized administrator of ScholarWorks@GVSU.

For more information, please contact scholarworks@gvsu.edu. 


\section{Photodissociation of ozone in the Hartley band: Product state and angular distributions}

George C. McBane, Luan T. Nguyen, and Reinhard Schinke

Citation: The Journal of Chemical Physics 133, 144312 (2010); doi: 10.1063/1.3491813

View online: https://doi.org/10.1063/1.3491813

View Table of Contents: http://aip.scitation.org/toc/jcp/133/14

Published by the American Institute of Physics

\section{Articles you may be interested in}

Photodissociation of ozone in the Hartley band: Potential energy surfaces, nonadiabatic couplings, and singlet/ triplet branching ratio

The Journal of Chemical Physics 132, 044305 (2010); 10.1063/1.3299249

Theoretical study of the ultraviolet photodissociation of ozone. Comparison with experiments

The Journal of Chemical Physics 101, 3806 (1994); 10.1063/1.467498

The photodissociation of N,N-dimethylnitrosamine at $355 \mathrm{~nm}$ : The effect of excited-state conformational changes on product vector correlations

The Journal of Chemical Physics 147, 013925 (2017); 10.1063/1.4982613

The photodissociation of ozone in the Hartley band: A theoretical analysis

The Journal of Chemical Physics 123, 074305 (2005); 10.1063/1.2001650

Theory of the photodissociation of ozone in the Hartley continuum: Potential energy surfaces, conical intersections, and photodissociation dynamics

The Journal of Chemical Physics 123, 014306 (2005); 10.1063/1.1903947

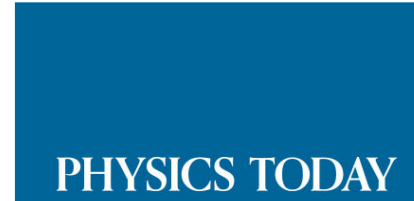

WHITEPAPERS
ADVANCED LIGHT CURE ADHESIVES

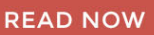

Take a closer look at what these environmentally friendly adhesive systems can do
PRESENTED BY

Q. MASTERBOND 


\title{
Photodissociation of ozone in the Hartley band: Product state and angular distributions
}

\author{
George C. McBane, ${ }^{1, a)}$ Luan T. Nguyen, ${ }^{1}$ and Reinhard Schinke ${ }^{2, b)}$ \\ ${ }^{1}$ Department of Chemistry, Grand Valley State University, Allendale, Michigan 49401, USA \\ ${ }^{2}$ Max-Planck-Institut für Dynamik und Selbstorganisation, D-37073 Göttingen, Germany
}

(Received 13 July 2010; accepted 2 September 2010; published online 12 October 2010)

\begin{abstract}
Product state properties from the photodissociation of ozone in the ultraviolet Hartley band are investigated by trajectory surface-hopping calculations. The diabatic B and R state potential energy and coupling surfaces of Schinke and McBane [J. Chem. Phys. 132, 044305 (2010)] are employed. The properties computed include rotational and vibrational distributions in both the singlet and triplet channels, the total internal energy distribution in the triplet channel, and the photodissociation anisotropy parameter $\beta$ in the singlet channel. A method for computing $\beta$ from trajectories computed in internal Jacobi coordinates is described. In the singlet channel, the vibrational distribution is in good agreement with the experimental results. The observed increase in $\beta$ with increasing photolysis wavelength is reproduced by the calculations and is attributed to the effects of the bending potential on the $\mathrm{B}$ state late in the fragmentation. The computed $\beta$ values are too high with respect to experiment, and the peaks $j_{\max }$ of the singlet-channel rotational distributions are too low; these discrepancies are attributed to a too steep bending potential at long $\mathrm{O}-\mathrm{O}$ distances. In the triplet channel, the main part of the internal energy distribution is described well by the calculations, although the detailed structures observed in the experiment are not reproduced. The experimental rotational distributions are well reproduced, although the maxima appear at slightly too high $j$. The triplet state product energy distributions are shown to depend largely on the distribution of hopping points onto the $\mathrm{R}$ state surface. A Landau-Zener model constructed as a function of the $\mathrm{O}_{2}$ bond distance provides a good physical description of the two-state dynamics. The high internal energy $\mathrm{O}_{2}$ products that cannot be attributed to the excitation of the Herzberg states remain unexplained. (C) 2010 American Institute of Physics. [doi:10.1063/1.3491813]
\end{abstract}

\section{INTRODUCTION}

The dissociation of ozone through absorption of ultraviolet light in the Huggins-Hartley band system is an important process in the atmosphere. ${ }^{1}$ It has been studied in detail both experimentally ${ }^{2,3}$ and theoretically. ${ }^{4}$ The Huggins and Hartley bands are due to the excitation of the same electronic state, namely, $3{ }^{1} A^{\prime}\left({ }^{1} B_{2}\right.$ in $\left.C_{2 v}\right)$, which has a relatively large transition dipole moment (TDM) $\mu_{\mathrm{XB}}$ of approximately $2 \mathrm{D}$ with the electronic ground state $1{ }^{1} A^{\prime}\left({ }^{1} A_{1}\right)$. The excited ozone dissociates mainly into the following two product channels:

$$
\begin{aligned}
\mathrm{O}_{3}\left(X^{1} A^{\prime}\right)+h \nu \rightarrow \mathrm{O}_{3}\left(3{ }^{1} A^{\prime}\right) & \rightarrow \mathrm{O}\left({ }^{3} P\right)+\mathrm{O}_{2}\left({ }^{3} \Sigma_{g}^{-}\right) \\
& \rightarrow \mathrm{O}\left({ }^{1} D\right)+\mathrm{O}_{2}\left({ }^{1} \Delta_{g}\right) .
\end{aligned}
$$

The dominant channel is the singlet channel (2), which diabatically correlates with $\mathrm{O}_{3}\left(3^{1} A^{\prime}\right)$ and accounts for about $92 \%$ of the quantum yield. Following Hay et al., ${ }^{5}$ we call the $3{ }^{1} A^{\prime}$ diabatic state the B state. The significant $(\sim 8 \%)$ population of the triplet channel (1), ${ }^{2,3}$ with both $\mathrm{O}$ and $\mathrm{O}_{2}$ in their ground electronic states, is due to a transition to a strongly repulsive state with ${ }^{1} A^{\prime}$ symmetry called the $\mathrm{R}$ state. Another

\footnotetext{
${ }^{a)}$ Electronic mail: mcbaneg@gvsu.edu.

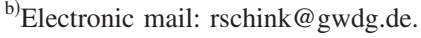

${ }^{1} A^{\prime}$ state, termed $\mathrm{A}$, is energetically close to $\mathrm{B}$ and $\mathrm{R}$ and dissociates, like the B state, into the singlet channel. However, its TDM with the ground state is about 100 times smaller than $\mu_{\mathrm{XB}}$ and therefore direct excitation of $\mathrm{A}$ can be neglected.

The electronic structure of ozone is complex and has been studied for more than three decades; for a list of references, see the recent review article of Grebenshchikov et al. ${ }^{4}$ Potential energy surfaces (PESs) describing the B state have been constructed by Hay et al., ${ }^{5}$ Sheppard and Walker, ${ }^{6}$ Yamashita et al., ${ }^{7}$ Banichevich et al., ${ }^{8}$ Baloïtcha and Balint-Kurti, ${ }^{9}$ and Qu et al. ${ }^{10}$ These PESs have supported investigations of the main features of the UV absorption and the fragmentation into the major channel (2). ${ }^{4}$ Since the triplet quantum yield is less than $10 \%$, it is a reasonable approximation to treat the singlet-channel dynamics using only the B state surface. However, a rigorous treatment of the dissociation requires more than just the $\mathrm{B}$ state.

Qu et al. ${ }^{10}$ constructed diabatic PESs for the excited states $\mathrm{B}, \mathrm{A}$, and $\mathrm{R}$, and the ground electronic state $\mathrm{X}$. Although $\mathrm{Qu}$ et al. did not determine the off-diagonal coupling potentials, these PESs revealed the crossing seams where transitions between the diabatic states, B and R or B and A, for example, would most likely occur. Then, Qu et al. ${ }^{11}$ applied a simple trajectory surface-hopping (TSH) method, us- 
ing an expression of Landau-Zener form with one adjustable parameter for the $\mathrm{B} / \mathrm{R}$ transition probability, to study the energy distributions in the triplet channel.

The work of $\mathrm{Qu}$ et al. ${ }^{10}$ was recently extended by Schinke and McBane. ${ }^{12}$ They constructed new diabatic PESs for states X, B, A, and R in the same manner as Qu et al. The electronic structure calculations were performed at a slightly more accurate level of theory and the grid of coordinates was significantly enlarged. Most importantly, coupling potentials between the different diabatic states $-V_{\mathrm{BR}}, V_{\mathrm{BA}}, V_{\mathrm{AX}}$, and $V_{\mathrm{AR}}$ - were also constructed. In the first application, the PESs and coupling potentials were used to calculate the quantum yield for the triplet channel by means of Tully's fewest-switches TSH method. ${ }^{13}$ A good agreement with the experimental data was obtained for wavelengths across the whole Hartley band from 220 to $310 \mathrm{~nm}$. The coupling function $V_{\mathrm{BR}}$ included one parameter, $\epsilon$, that controlled the width of the coupling region around the $\mathrm{B} / \mathrm{R}$ crossing seam, but its value affected the quantum yields only modestly. A comparison with quantum mechanical calculations, independently performed with the same potentials by Grebenshchikov, confirmed that the TSH method is trustworthy for describing the UV photodissociation of ozone.

The present work is a continuation of Ref. 12. We investigate the distribution of the excess energy into translational, vibrational, and rotational motion of the products, in both the singlet and the triplet channels, using the TSH approach. In addition, we calculate the anisotropy parameter $\beta$ for the singlet channel. We compare our computed quantities with detailed experimental results. Accompanying quantum calculations by Grebenshchikov will be published separately.

\section{DYNAMICS CALCULATIONS}

Ozone dissociation in the ultraviolet is a fast and direct process involving high kinetic energies, so classical mechanics should provide an adequate description of its nuclear dynamics. The electronic surfaces are well separated for most nuclear arrangements, and the molecule spends only a small amount of time near their crossings, so a surface-hopping description of the electronic transitions should also be adequate. Our classical surface-hopping calculations for total angular momentum $J=0$ were performed as described in Ref. 12. The calculations used symmetric Jacobi coordinates $R$ (distance from the central $\mathrm{O}$ atom to the center of mass of the two end atoms), $r$ (distance between the two end atoms), and $\gamma$ (angle between $\boldsymbol{R}$ and $\boldsymbol{r}$ ). Trajectories were started on the B state PES with coordinates and momenta initially selected from Gaussian distributions in the six-dimensional phase space of Jacobi coordinates and momenta chosen to model the ground state vibrational wavefunction of ozone. ${ }^{14}$ For a given excitation wavelength, the initially chosen initial conditions were then subjected to an energy filter; only phase space points within $0.01 \mathrm{eV}$ of the correct total energy were used. Trajectories were propagated to a product separation distance of at least $6 \AA$.

The calculations included two diabatic surfaces, B and $\mathrm{R}$, using the fewest-switches method of Tully. ${ }^{13}$ Our implementation was described in Ref. 12. In that work, we used a simplified method for energy conservation at hops that divided the necessary change in kinetic energy equally among the three Jacobi momenta. During the present work, we found that that method introduced artifacts into the triplet product rotational state distributions. We therefore switched to using the " $\mathrm{g}$ prescription" originally described by Blais and Truhlar ${ }^{15}$ and later reviewed by Hack et al. ${ }^{16}$ In this approach, the momenta are adjusted at a hop along a direction given by the gradient of the difference in potential between the initial and the final electronic states. This change in method affected only the computed rotational state distributions for the triplet products; other computed quantities, including the triplet quantum yield and the vibrational distributions, were essentially unaffected.

\section{A. Product state distributions}

In the computation of final state distributions, the contribution of each trajectory was weighted by the square of its initial transition dipole moment, $\left|\mu_{\mathrm{XB}}\right|^{2}$, to account for the variation in the absorption probability with the initial molecular geometry. The transition dipole moments were obtained from spline fits to the tabulated transition moment vector components of Baloïtcha and Balint-Kurti. ${ }^{9}$

For each trajectory, we extracted the final translational and rotational energies from time derivatives of Jacobi coordinates $R^{\prime}$ and $\gamma^{\prime}$ appropriate for the product channel and found the vibrational energy $E_{\mathrm{vib}}$ from the conservation of energy. To assign vibrational quantum numbers, we solved the equation $E_{\mathrm{vib}}=\omega_{e}\left(v_{\mathrm{cl}}+1 / 2\right)-\omega_{e} x_{e}\left(v_{\mathrm{cl}}+1 / 2\right)^{2}$ to assign a "classical vibrational quantum number" $v_{\mathrm{cl}}$ using the $\omega_{e}$ and $\omega_{e} x_{e}$ molecular constants of Nieh and Valentini ${ }^{17}$ for $\mathrm{O}_{2}\left(a^{1} \Delta_{g}\right)$ and those of Laher and Gilmore ${ }^{18}$ for $\mathrm{O}_{2}\left(X^{3} \Sigma_{g}^{-}\right)$. Then, we assigned each trajectory to the vibrational bin corresponding to the integer $v$ nearest to $v_{\mathrm{cl}}$. We followed a similar binning procedure for assigning rotational quantum numbers using $B_{e}$ and $\alpha_{e}$ molecular constants from the same sources. The ground state of oxygen has only odd rotational levels, so trajectories ending there were assigned to the nearest odd value of $j$.

For the vibrational distribution in the singlet channel, we also tested the Gaussian binning method ${ }^{19,20}$ of Bonnet and Rayez using a Gaussian weighting function proportional to $\exp \left(-\left(v_{\mathrm{cl}}-v\right)^{2} / 2 \sigma^{2}\right)$ with a standard deviation $\sigma$ of 0.05 . It yielded essentially the same results as standard binning, with some differences in details as described below.

\section{B. Angular distribution calculations}

The anisotropy parameter $\beta$ describes the distribution of product travel directions with respect to the polarization direction of the dissociation light. The limiting values for onephoton dissociation are $\beta=2$ for fragments traveling as closely as possible along the photolysis polarization and $\beta$ $=-1$ for fragments perpendicular to the photolysis polarization. For a one-photon dissociation, Zare $^{21}$ showed that $\beta$ may be computed from an average over $\theta_{\mathrm{m}}$, the angle between the final velocity vector of the $\mathrm{O}$ atom product and the 
initial transition dipole vector of ozone at the time of photoabsorption, by $\beta=2\left\langle P_{2}\left(\cos \theta_{\mathrm{m}}\right)\right\rangle$, where $P_{2}(x)$ is a Legendre polynomial.

Our trajectories were run in internal coordinates. The extraction of $\theta_{\mathrm{m}}$ from a trajectory record requires two additional pieces of information: the direction of the transition dipole moment vector for the initial configuration of ozone and the knowledge of the rotation of the internal coordinate system in the laboratory frame during the trajectory. We obtained the components of the transition dipole moment vector from spline fits of the TDM listings of Baloïtcha and Balint-Kurti. $^{9}$

To find the relation of the internal (Jacobi) and spacefixed frames during the trajectory, we applied the conservation of angular momentum in the form given by Demyanenko et al. $^{22}$ Since the total angular momentum is zero, any rotation of $\boldsymbol{r}$ in the space-fixed frame must be accompanied by a compensating rotation of $\boldsymbol{R}$. If the orientations of the $\boldsymbol{r}$ and $\boldsymbol{R}$ vectors in the space-fixed frame are given by angles $\phi_{r}$ and $\phi_{R}$, and the Jacobi angle $\gamma=\phi_{r}-\phi_{R}$, then for $J=0$, we have

$$
\frac{d \phi_{R}}{d t}=-\left(1+\frac{\mu R^{2}}{\mu_{\mathrm{O}_{2}} r^{2}}\right)^{-1} \frac{d \gamma}{d t},
$$

where $\mu$ is the $\mathrm{O}-\mathrm{O}_{2}$ reduced mass and $\mu_{\mathrm{O}_{2}}$ is the $\mathrm{O}_{2}$ reduced mass. We chose the space-fixed frame so that its $z$ axis lies along the direction of $\boldsymbol{R}$ at the beginning of the trajectory. We then added Eq. (3) to the set of coupled differential equations being solved by the trajectory program, setting $\phi_{R}=0$ at the beginning of each trajectory. From the values of the Jacobi coordinates and momenta and $\phi_{R}$ at the termination of each trajectory, we constructed the final velocity components of the $\mathrm{O}$ atom in the space frame as described in the Appendix and computed the angle $\theta_{\mathrm{m}}$ between the final velocity and the initial transition dipole moment. $\beta$ was computed by averaging $2 P_{2}\left(\cos \theta_{\mathrm{m}}\right)$ over the set of trajectories using $\left|\mu_{\mathrm{XB}}\right|^{2}$ as a weighting factor.

\section{RESULTS AND DISCUSSION}

\section{A. Singlet channel}

\section{Vibrational distribution of $\mathrm{O}_{2}\left(a^{1} \Delta_{g}\right)$}

$\mathrm{Qu}$ et al. ${ }^{10}$ presented comparisons of their vibrational distributions with the experimental results of Dylewski et $a .^{23}$ and reviewed earlier calculations. Our results, shown in Fig. 1, are similar. The agreement with the experiment is quite good, with the exception that at $235 \mathrm{~nm}$ the drop from $v=0$ to 1 is steeper in the computed distributions than in the experiment. Both the experimental and the computed vibrational populations decrease monotonically from $v=0$ at all wavelengths. This property of the distribution appears to be sensitive to the shape of the B state potential surface since several earlier calculations ${ }^{6,9,24}$ on other surfaces found maxima at $v=1$.

In the computed distributions for most wavelengths, a small population (3\% or less) appears in the first energetically closed channel. Several factors contribute to this spurious population. First, the B state asymptotic energy is too
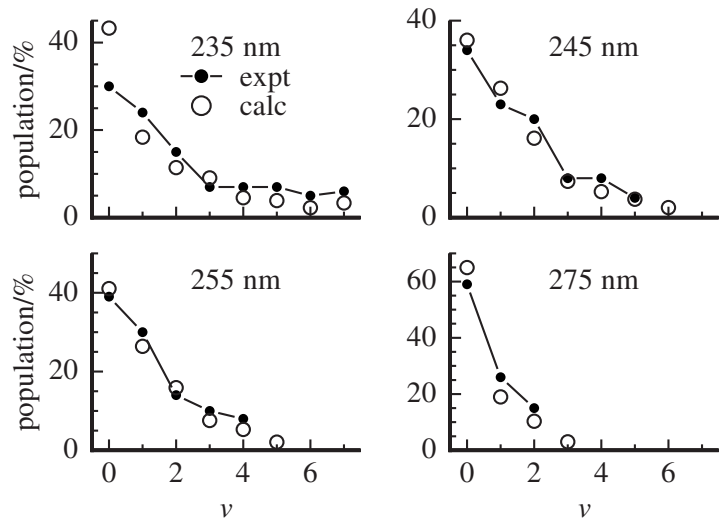

FIG. 1. Vibrational distributions of $\mathrm{O}_{2}\left(a{ }^{1} \Delta_{g}\right)$. The experimental data are from Dylewski et al. (Ref. 23).

low by $0.067 \mathrm{eV}$, and our energy window for selection of initial conditions had a half-width of $0.010 \mathrm{eV}$, so the outgoing trajectories can have a total available energy that is too high by as much as $0.077 \mathrm{eV}$ or $621 \mathrm{~cm}^{-1}$. Second, the binning procedure assigns each trajectory to the vibrational bin with $v$ closest to $v_{\mathrm{cl}}$ and occasionally "promotes" a trajectory to a closed vibrational state. The Gaussian binning procedure $^{19,20}$ sometimes helps with this second effect; for some wavelengths (not displayed in Fig. 1), the Gaussian binning procedure reduced the spurious population by factors of 2-3. For the wavelengths shown, the Gaussian and standard binning procedures gave very similar results.

\section{Rotational distribution of $\mathrm{O}_{2}\left(a^{1} \Delta_{g}\right)$}

Valentini et al. ${ }^{25}$ measured the rotational and vibrational distributions of the $\mathrm{O}_{2}\left(a^{1} \Delta_{g}\right)$ product using coherent antiStokes Raman spectroscopy. We have extracted the corresponding rotational distributions in the singlet channel from trajectories at 240 and $266 \mathrm{~nm}$. Representative results for three vibrational states at each wavelength are shown in Fig. 2. In that figure, all the distributions for each wavelength

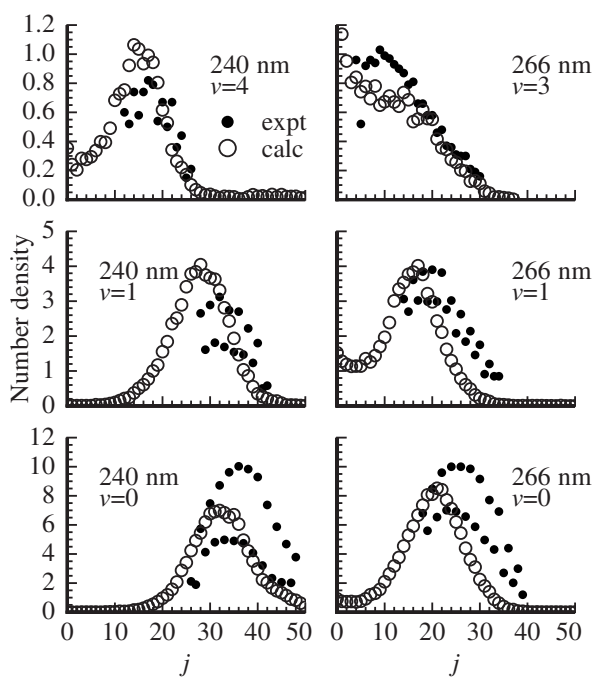

FIG. 2. Rotational distributions of $\mathrm{O}_{2}\left(a^{1} \Delta_{g}\right)$. The experimental data are from Valentini et al. (Ref. 25). The "number density" vertical scale is taken from the experimental paper. A single scaling factor is used for each wavelength, chosen for $v=0$. 
TABLE I. Anisotropy parameter $\beta$ in the singlet channel from experiment (Ref. 23) and from the trajectory calculations (QCT). The "avg" column for the experimental rows does not list the average values given by Table II of Dylewski et al. but instead a weighted average computed from their vibrational state populations.

\begin{tabular}{clllllllll}
\hline \hline $\begin{array}{c}\lambda \\
(\mathrm{nm})\end{array}$ & & $v=0$ & $v=1$ & $v=2$ & $v=3$ & $v=4$ & $v=5$ & $v=6$ & Avg \\
\hline \multirow{2}{*}{235} & Expt. & 1.1 & 1.19 & 1.21 & 1.15 & 1 & 0.84 & 0.57 & 1.08 \\
& QCT & 1.36 & 1.36 & 1.36 & 1.35 & 1.38 & 1.42 & 1.56 & 1.36 \\
245 & Expt. & 1.3 & 1.28 & 1.23 & 1.15 & 1.08 & 1.03 & & 1.20 \\
& QCT & 1.42 & 1.4 & 1.38 & 1.41 & 1.41 & 1.54 & 1.67 & 1.42 \\
255 & Expt. & 1.2 & 1.33 & 1.28 & 1.35 & 1.32 & & & 1.29 \\
& QCT & 1.50 & 1.45 & 1.48 & 1.48 & 1.58 & & & 1.48 \\
265 & Expt. & 1.5 & 1.46 & 1.27 & 1.37 & & & & 1.44 \\
& QCT & 1.51 & 1.49 & 1.57 & 1.71 & 1.78 & & & 1.55 \\
275 & Expt. & 1.6 & 1.5 & 1.5 & & & & & 1.56 \\
& QCT & 1.56 & 1.62 & 1.73 & 1.77 & & & & 1.61 \\
285 & Expt. & 1.8 & 1.6 & & & & & & 1.74 \\
& QCT & 1.73 & 1.82 & 1.83 & & & & & 1.76 \\
\hline \hline
\end{tabular}

have been scaled by a single factor, chosen to make the trajectory results approximately match the experimental scale for $v=0$.

The measured rotational distributions are sharply peaked at relatively high $j$, and the peaks move to lower $j$ as the vibrational quantum number increases. The trajectory results capture the shapes of the distributions and the variation in the peak positions with $v$ very well. The computed rotational distributions peak at lower rotational quantum numbers than the experimental ones, especially at low $v$. We discuss a possible origin for this disagreement at the end of Sec. III A 3.

The results of $\mathrm{Qu}$ et al. ${ }^{10}$ are in better agreement with the experimental distributions. Baloïtcha and Balint-Kurti, ${ }^{9}$ on the other hand, reported rotational distributions for $266 \mathrm{~nm}$ whose peaks are at slightly higher $j$ than the experimental ones. This comparison of results from three different calculations shows the sensitivity of the rotational distribution to the details of the angle dependence of the B state PES.

In the experimental distributions, states with even $j$ have higher populations than the odd ones. Valentini et al. identified the even-odd alternation as the result of preferential coupling of the odd states to the triplet channel. That preference is not present in our calculations, so the trajectory results should represent averages of the even and odd experimental curves. That is approximately what happens, although in Fig. 2 , a modest disagreement in the vibrational populations at $240 \mathrm{~nm}$ makes the computed rotational distributions for $v$ $>0$ look too high.

\section{Angular distribution of $\mathrm{O}\left({ }^{1} D\right)$}

Dylewski et $a .^{23}$ measured the photodissociation anisotropy parameter $\beta$ for the $\mathrm{O}\left({ }^{1} D\right)$ product at wavelengths between 235 and $305 \mathrm{~nm}$ using supersonically cooled ozone. They found that at the shortest wavelengths $\beta \sim 1.2$, but that $\beta$ increased as the photolysis wavelength increased, reaching $\beta \sim 1.8$ at $298 \mathrm{~nm}$.

In the Hartley band, the transition dipole moment in $C_{2 v}$ geometry lies parallel to a line connecting the two end atoms. If the departing $\mathrm{O}$ atom traveled directly along the direction of the breaking $\mathrm{O}-\mathrm{O}$ bond at the moment of absorption, justifying the "axial recoil" model, and the absorption occurred from the ground state equilibrium geometry, a value of $\beta$ $=1.18$ would be expected. (Loss of the central atom is very unlikely; for example, at $226 \mathrm{~nm}$, only $0.01 \%$ of the trajectories followed that route.) The experimental observation that $1.2 \leq \beta \leq 1.8$ indicates that the outgoing atoms are traveling more nearly along the photolysis polarization direction than the axial recoil model predicts.

The observed increase of $\beta$ with increasing $\lambda$ contrasts with the common expectation that as photolysis energies decrease, the excited complex lifetime will increase, the internal or rotational motion of the complex will reduce the correlation of the bond directions with the photolysis polarization, and $\beta$ will decrease. Dylewski et al. discussed their measurements of $\beta$ in terms of the bond angle "at the time of dissociation." They pointed out that their observations implied a widening of the bond angle and attributed it to "significant activity in the bending and stretching modes during the photodissociation process."

Dylewski et al. reported $v$-specific $\beta$ values measured at the peaks of the intensity distribution for each vibrational level and average $\beta$ values determined by fitting whole images that included the contributions from all $v$. The increase in $\beta$ with increasing wavelength is clear in the $v$-specific values and in a weighted average of those values constructed using the measured vibrational populations. However, the overall average $\beta$ values of Dylewski et al. are lower and do not show a smooth trend with $v$. We have focused on the $v$-specific results, and we also report the weighted average in the last column of Table I.

We computed $\beta$ for most of the wavelengths used by Dylewski et al. The results appear in Table I and Fig. 3. The increase in $\beta$ with increasing photolysis wavelength is reproduced well in the calculations, although the computed $\beta$ values are generally higher than the measured ones. The disagreement is $0.2-0.3$ at most wavelengths but decreases at $285 \mathrm{~nm}$. $\beta$ has no clear trend with $v$ for a single wavelength in either experiment or calculation; the computed trend is 


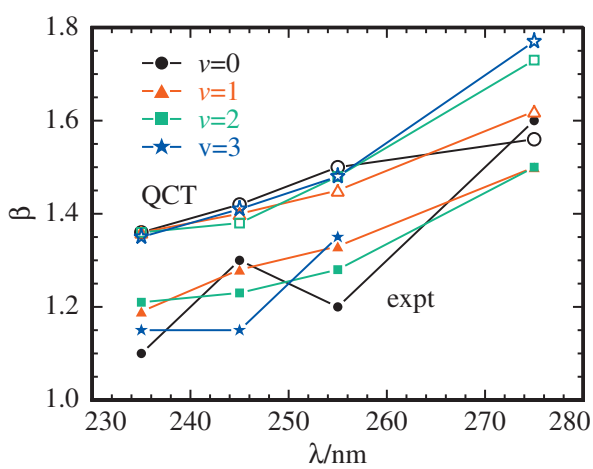

FIG. 3. Experimental (solid symbols) and computed (open symbols) $\beta$ values at different wavelengths for $v=0-3$, from Table I. Values for the same $v$ at each wavelength are connected by lines to help make the overall trends clear.

very weak and the experimental error estimate of $10 \%$ is wide enough that error bars for most vibrational levels at each wavelength overlap.

The increase of $\beta$ with increasing wavelength, seen in both experiment and calculation, can be traced to a clear physical origin. During the initial moments of the photodissociation, the departing atom and the central atom move apart, while the third atom is approximately stationary, in agreement with a simple impulsive picture. This motion causes a decrease of the bond angle. The initial dissociation therefore excites the bending motion of ozone on the B state, starting near $\alpha=117^{\circ}$ and moving toward smaller bond angles.

Figure 4 shows an angular contour plot of the B state along with two trajectories computed for the $255 \mathrm{~nm}$ dissociation: one typical and one extreme. The angular minimum in the B state well lies near $\alpha=107^{\circ}$ and does not vary much with the bond lengths. The angular potential has little influence on the direction of the departing atom during the initial part of the trajectory since the motion is nearly along the direction of the bending force. Typical trajectories pass through the angular minimum when the length of the breaking bond is about $r_{1} \sim 3 a_{0}$; beyond $r_{1} \sim 4 a_{0}$, the trajectories are on the small-angle side of the bending potential and moving more nearly parallel to the angular contour lines. Here, they feel a force that tends to deflect the departing atoms away from axial recoil toward the direction of the original transition dipole moment and increase the value of $\beta$. The angular potential is relatively weak for bond lengths of $4 a_{0}$ and longer, and for most trajectories, $d \alpha / d t$ remains negative during the whole dissociation. That is the case for the typical trajectory (a) in Fig. 4. Nonetheless, the departing atom is deflected away from the axial recoil by the inner wall of the bending potential. Trajectory (b) in Fig. 4 is so strongly deflected from the inner side of the bending potential that $\alpha$ begins increasing again: the second half of a bending vibration has begun, and the rotation direction of the product $\mathrm{O}_{2}$ actually reverses. Only a small fraction of the trajectories shows this behavior; this example was not chosen as representative but to highlight the effect of the bending potential even when the bond is nearly broken.

In the lower panels of Fig. 4, the trajectories of all three atoms are plotted in space-fixed coordinates. The right-hand
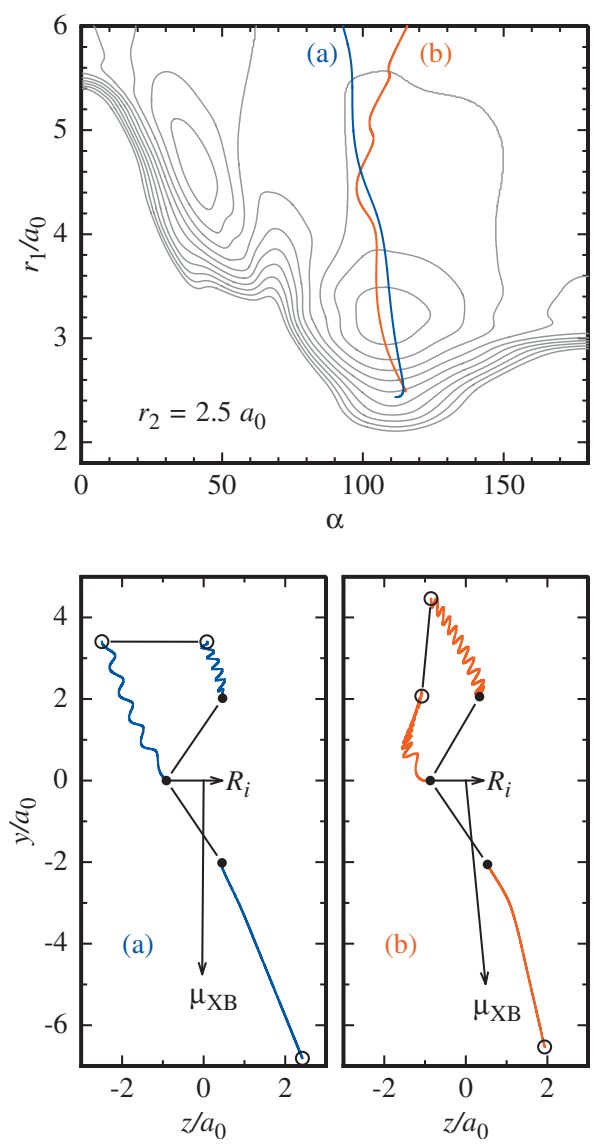

FIG. 4. A "typical" (a) and an "extreme" (b) trajectory, both computed for dissociation wavelength of $255 \mathrm{~nm}$, showing the influence of the B-state bending potential on $\theta_{\mathrm{m}}$. Upper panel: $r_{1}-\alpha$ potential contours of the $\mathrm{B}$ state computed for $r_{2}=2.5 a_{0}$. The contour interval is $0.2 \mathrm{eV}$ and the lowest contour plotted is $3.85 \mathrm{eV}$. Trajectory (a), which ends in $j=14$, is typical; trajectory (b), which ends in $j=8$, highlights the effect of the bending potential at large $r_{1}$. Lower panels: atomic paths for those two trajectories in spacefixed coordinates. The initial atomic positions are shown with solid dots and final atomic positions with circles. The direction of the transition dipole moment vector is indicated by an arrow that intersects the initial $R$ vector at the center of mass.

turn made by the departing atom in trajectory (b) in the lower right is clear. The outgoing atom for trajectory (a) is similarly deflected in the region $-3 a_{0} \geq y \geq-4 a_{0}$, but the deflection is much smaller. In both cases, the departing atom turns toward the direction of the TDM, and thus to smaller $\theta_{\mathrm{m}}$ and larger $\beta$.

The axial recoil picture thus applies accurately to the initial stage of the dissociation, but the departing products scatter from the inner wall of the bending potential late in the fragmentation. Trajectories at longer dissociation wavelengths have lower kinetic energies and are more strongly influenced by the weak bending potential. Their departing atoms therefore are deflected more, and their $\beta$ values are correspondingly higher, than trajectories from dissociation at shorter wavelengths.

A stronger bending potential at long $\mathrm{O}-\mathrm{O}_{2}$ distances leads to a higher $\beta$ and a lower $j$ for the products. The computed $\beta$ values are too high compared with experiment, and the rotational distributions shown in the preceding section peak at rotational quantum numbers that are too low. We 


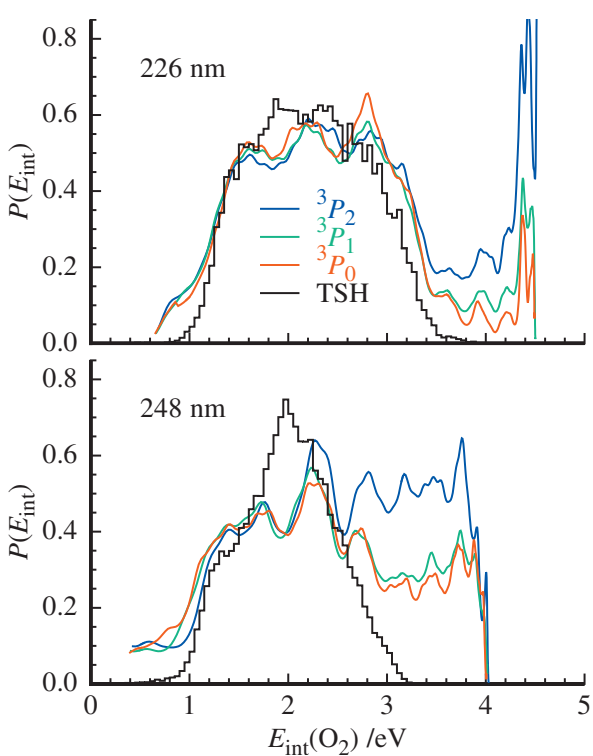

FIG. 5. Experimental and computed internal energy distributions in the triplet channel for 248 and $226 \mathrm{~nm}$. Experimental distributions are those from Figs. 4 and 5 of Ref. 26 after transformation to an internal energy axis. The curves are all separately normalized so that the part of each one with $E_{\text {int }}<2.5 \mathrm{eV}$ encloses the same area.

conclude that on the $a b$ initio $\mathrm{B}$ state surface, the bending potential is slightly too strong for long $\mathrm{O}-\mathrm{O}_{2}$ distances.

\section{B. Triplet channel}

\section{Internal versus translational energy partitioning}

Most products produced in the triplet channel with $E_{\text {int }}$ $<2.5 \mathrm{eV}$ come from transitions from the initially excited $\mathrm{B}$ state to the repulsive $\mathrm{R}$ state. The $\mathrm{B} / \mathrm{R}$ crossing seam lies near a bond length of $3.3 a_{0}-3.5 a_{0}$ for the breaking bond, and its position is roughly independent of the remaining $\mathrm{O}_{2}$ bond length, as shown in Fig. 6 of Ref. 12 and in Fig. 6 below. Qu et al. discussed the energy distribution in the triplet-channel products on the basis of an earlier set of diabatic surfaces ${ }^{10}$ and a simple surface-hopping model. ${ }^{11}$ They showed that the dependence of the transition probability at the crossing seam on the length of the $\mathrm{O}_{2}$ bond had a strong influence on the final vibrational and translational distributions. Here, we extend that analysis with the new potential and coupling surfaces and the more general fewest-switches approach.

Figure 5 shows the $\mathrm{R}$ state internal energy distributions from surface-hopping calculations at 226 and $248 \mathrm{~nm}$ overlaid on the experimental results of Brouard et al. ${ }^{26}$ Each panel displays the experimental distributions obtained from all three spin-orbit states of $\mathrm{O}\left({ }^{3} P\right)$. The trajectory calculations do not include spin-orbit splitting, so only a single theoretical histogram appears in each panel.

The experimental distributions for the three spin-orbit states are very similar for $E_{\text {int }}<2.5 \mathrm{eV}$ at $248 \mathrm{~nm}$ and for the whole distribution at $226 \mathrm{~nm}$. At $226 \mathrm{~nm}$, the sharp peak in the distribution at $E_{\text {int }}>4.3 \mathrm{eV}$ is associated ${ }^{27}$ with the production of electronically excited $\mathrm{O}_{2}$ in the Herzberg states $A^{3} \Sigma_{u}^{+}$and $A^{\prime}{ }^{3} \Delta_{u}$. That channel has a threshold at $234.1 \mathrm{~nm}$ and is unavailable at longer dissociation wavelengths. The part of the $226 \mathrm{~nm}$ distribution between 3.6 and $4.3 \mathrm{eV}$, and the entire $248 \mathrm{~nm}$ distribution above $3.2 \mathrm{eV}$, arise from a mechanism that is not modeled by our $\mathrm{B} \rightarrow \mathrm{R}$ calculations. In Ref. 12, Schinke and McBane argued that the $\mathrm{B} \rightarrow \mathrm{A} \rightarrow \mathrm{X}$ mechanism proposed by Brouard et al. ${ }^{26}$ was also not a viable explanation because the $\mathrm{B} \rightarrow \mathrm{A}$ and $\mathrm{A} \rightarrow \mathrm{X}$ transition probabilities were too small. The origin of that group of slow product molecules therefore remains unknown.

The TSH calculations reproduce the observed $E_{\text {int }}$ distribution for the $226 \mathrm{~nm}$ dissociation fairly well. The overall shape, with steep sides and a flat top, is well reproduced. The width of the calculated distribution is slightly smaller than the observed width. The experimental structure consists of three maxima separated by minima that are lower by about $10 \%$. The less well defined structure in the computed distribution is sensitive to small changes in the B state potential surface and we cannot claim that the modest agreement arises from an accurate physical model.

For the $248 \mathrm{~nm}$ dissociation, the comparison between experiment and theory is less clear-cut because of the variation in the experimental distributions for different spin-orbit states and because the high-energy shoulder in $P\left(E_{\text {int }}\right)$ our calculations do not model is more important. The shoulder is much larger for $\mathrm{O}\left({ }^{3} P_{2}\right)$ than for the two higher spin-orbit states. The low-energy side of the distribution is reproduced well by the calculation. The experimental distribution is less flat-topped at 248 than at $226 \mathrm{~nm}$, and the same is even more true of the computed distribution.

We now explore the physical origins of the computed $P\left(E_{\text {int }}\right)$ distributions. Although the PESs are symmetric in the two bond lengths, throughout this section we use $r_{1}$ for the length of the breaking bond, $r_{2}$ for the length of the surviving bond, and $r_{2 x}$ for the $\mathrm{O}_{2}$ bond length at the time the $\mathrm{B} / \mathrm{R}$ seam is crossed. The $\mathrm{B} / \mathrm{R}$ seam lies between 2.4 and $4 \mathrm{eV}$ above the asymptote of the triplet channel for most values of $\alpha$ and $r_{2}$. Therefore, the energy disposal in the triplet channel is strongly influenced by the dynamics occurring on the $\mathrm{R}$ state. To establish the extent of this influence, we started a set of trajectories on the $\mathrm{R}$ state at the crossing seam with no initial momentum, $\alpha=100^{\circ}$, and 150 different values of the $\mathrm{O}_{2}$ bond length $r_{2 x}$. The top panel of Fig. 6 shows the trajectories from five of these initial conditions superimposed on contours of the $\mathrm{R}$ state surface. The middle panel shows vibrational, rotational, and total internal energies of the resulting products as a function of $r_{2 x}$. The final rotational energy does not depend strongly on $r_{2 x}$. The product vibrational energy is a strong function of $r_{2 x}$, as one would predict from the R state contours; the partitioning of the available energy between product vibration and translation roughly reflects the potential energy on the $\mathrm{R}$ surface along the crossing seam. The plotted points are the final internal energies of a subset of triplet-channel trajectories from the main calculation at $226 \mathrm{~nm}$. They cluster closely around the curve based on the R-state-only model. There is a slight but systematic difference between the plotted points and the R-state-only model: on the average, trajectories with $r_{2 x}<2.9 a_{0}$ tend to have a little more internal energy than expected, while the opposite is true for trajectories crossing at higher $r_{2 x}$. We conclude that the main influence of the initial conditions and 

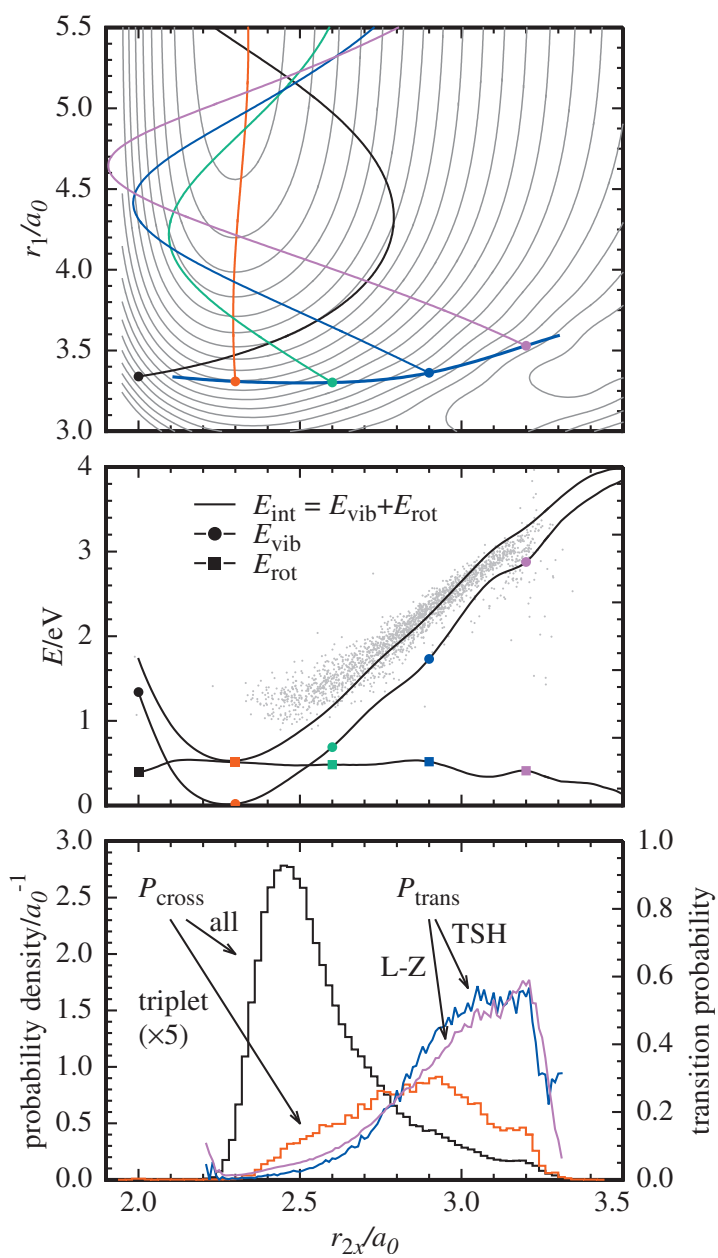

FIG. 6. Influence of crossing point along B/R seam on triplet product energy distributions. Top panel: R state contours for $\alpha=100^{\circ}$, and several sample trajectories started on the $\mathrm{R}$ surface at the $\mathrm{B} / \mathrm{R}$ seam without initial momentum. The $\mathrm{B} / \mathrm{R}$ crossing seam is indicated by the blue curve near $r_{1}=3.3 a_{0}$. The $r_{1}$ (vertical) axis is compressed by a factor of 2 compared to the $r_{2}$ axis. Middle panel: product vibrational, rotational, and total internal energies for trajectories started at the seam as a function of the initial $r_{2}$. The curves were constructed from 150 trajectories with different starting points along the seam; the five shown in the upper panel are marked. The gray points represent the final internal energies of 2000 triplet-channel TSH trajectories started on the B state with initial conditions corresponding to $226 \mathrm{~nm}$, plotted against $r_{2 x}$. Bottom panel: seam crossing-point distributions $P_{\text {cross }}\left(r_{2 x}\right)$ for all trajectories and for triplet-channel trajectories (scaled vertically by a factor of 5), and transition probabilities $P_{\text {trans }}\left(r_{2 x}\right)$ extracted from the TSH calculations and from an $r_{2 x}$-dependent Landau-Zener model. The transition probabilities are plotted against the right-hand axis.

the B state dynamics on the triplet products is in their influence on the distribution of seam crossing points and transition probabilities.

We next seek an understanding of the distribution of seam crossing positions for the triplet trajectories. This distribution must be the product of the distribution of seam crossing positions for all trajectories and a position-andmomentum-dependent transition probability, as outlined in Ref. 12. The crossing-point distributions at $226 \mathrm{~nm}$ for all trajectories and for triplet-channel trajectories are shown at the bottom panel of Fig. 6. The distribution for all trajectories is determined by the selection of initial conditions for the trajectories and the subsequent dynamics on the B state. It is strongly peaked near $r_{2 x}=2.45 a_{0}$ with a long tail extend- ing to higher $r_{2 x}$. The $r_{2 x}$ dependent transition probability $P_{\text {trans }}\left(r_{2 x}\right)$, which can be constructed from the trajectories by dividing the number of triplet-channel trajectories within an $r_{2 x}$ bin by the total number of trajectories in that bin, is also plotted there. It rises steeply from near zero around $r_{2 x}$ $=2.4 a_{0}$ to a broad maximum between $3.0 a_{0}$ and $3.2 a_{0}$, and then falls rapidly. In other words, trajectories crossing the seam with large values of the $\mathrm{O}_{2}$ bond length have much higher probabilities of switching to the $\mathrm{R}$ state. This important characteristic of the transition probability was pointed out by $\mathrm{Qu}$ et al. on the basis of a simple surface-hopping model. ${ }^{11}$ Here, we can explore it more fully. Within each $r_{2 x}$ bin, we have evaluated an average Landau-Zener transition probability according to

$$
P_{\text {trans, LZ }}\left(r_{2 x}\right)=1-\exp \left[-\frac{2 \pi}{\hbar} V_{\mathrm{BR}}^{2}\left(\frac{d \Delta V}{d r_{1}} \dot{r}_{1}\right)^{-1}\right] \text {. }
$$

Here, $V_{\mathrm{BR}}$ is the $\mathrm{B} / \mathrm{R}$ coupling matrix element, $d \Delta V / d r_{1}$ is the difference in the slopes of the $\mathrm{B}$ and $\mathrm{R}$ surfaces at the seam for a given $r_{2 x}$ and bond angle, and $\dot{r}_{1}$ is the velocity. While the slope difference and the velocity should strictly be evaluated along a direction perpendicular to the seam, since the seam is nearly perpendicular to the $r_{1}$ axis, we have approximated them with the simpler derivatives with respect to $r_{1}$. The resulting transition probability is also plotted in Fig. 6. It follows the corresponding curve from the TSH calculation remarkably well.

All three variables of the Landau-Zener model-the coupling strength $V_{\mathrm{BR}}$, the speed $\dot{r}_{1}$ perpendicular to the seam, and the difference in the slopes of the two surfaces $d \Delta V / d r_{1}$-contribute to the steep dependence of the hopping probability on $r_{2 x}$. The velocity is smaller for trajectories crossing the seam with large $r_{2}$ since more energy is taken up in potential energy on the B state. At large $r_{2}$, the B and R state slopes become more similar, and $V_{\mathrm{BR}}$ increases. All of those changes imply a more adiabatic behavior and therefore a larger B/R transition probability.

As the dissociation wavelength increases, the trajectories begin lower on the $\mathrm{B}$ state and cross the $\mathrm{B} / \mathrm{R}$ seam with shorter $\mathrm{O}_{2}$ bond lengths. The overall crossing-point distribution and the transition probability distribution therefore shift to a smaller $r_{2}$ in Fig. 6 , so the distribution of crossing points for triplet trajectories also shifts to smaller $r_{2}$ and the R-stateonly model suggests that the products should have lower vibrational energies.

\section{Vibrational distribution}

The computed triplet state vibrational distributions for several dissociation wavelengths are shown in Fig. 7. The distributions are broad, but do not extend all the way to the energetic limit. As the wavelength increases, the peak of the distribution shifts to lower vibrational quantum numbers, as expected. No state-resolved vibrational distribution measurements are available for comparison.

\section{Rotational distribution of $\mathrm{O}_{2}\left(X^{3} \Sigma_{g}^{-}\right)$}

Daniels and Wiesenfeld ${ }^{28}$ measured the rotational distributions of the product $\mathrm{O}_{2}\left(X^{3} \Sigma_{g}^{-}\right)$from the $248 \mathrm{~nm}$ photodis- 

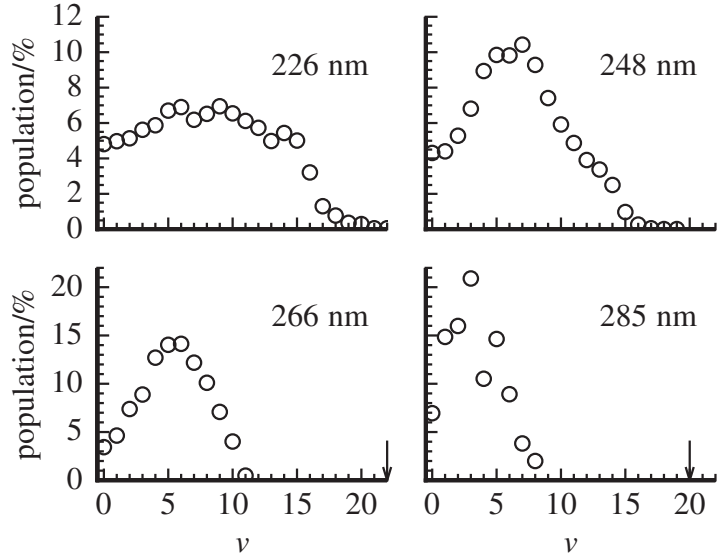

FIG. 7. Computed vibrational distributions in the triplet channel for several wavelengths. The small vertical arrows in the lower two panels indicate the maximum energetically allowed quantum numbers; for 226 and $248 \mathrm{~nm}$, the energetic limits are $v=29$ and 25 , respectively.

sociation of a room-temperature ozone sample in vibrational levels $v=9,12$, and 15 by laser induced fluorescence in the Schumann-Runge band. Spectroscopic limitations restricted their observations to $j \leq 55$. Figure 8 displays their measured distributions together with rotational distributions from our TSH calculations. Because the rotational and vibrational distributions are broad in the triplet channel, and the quantum yield for triplet is less than $10 \%, 10^{6}$ trajectories were needed to obtain adequate numbers of trajectories in the $(v, j)$ channels shown.

Both the observed and the computed rotational distributions are peaked at high rotational quantum numbers, as ex-
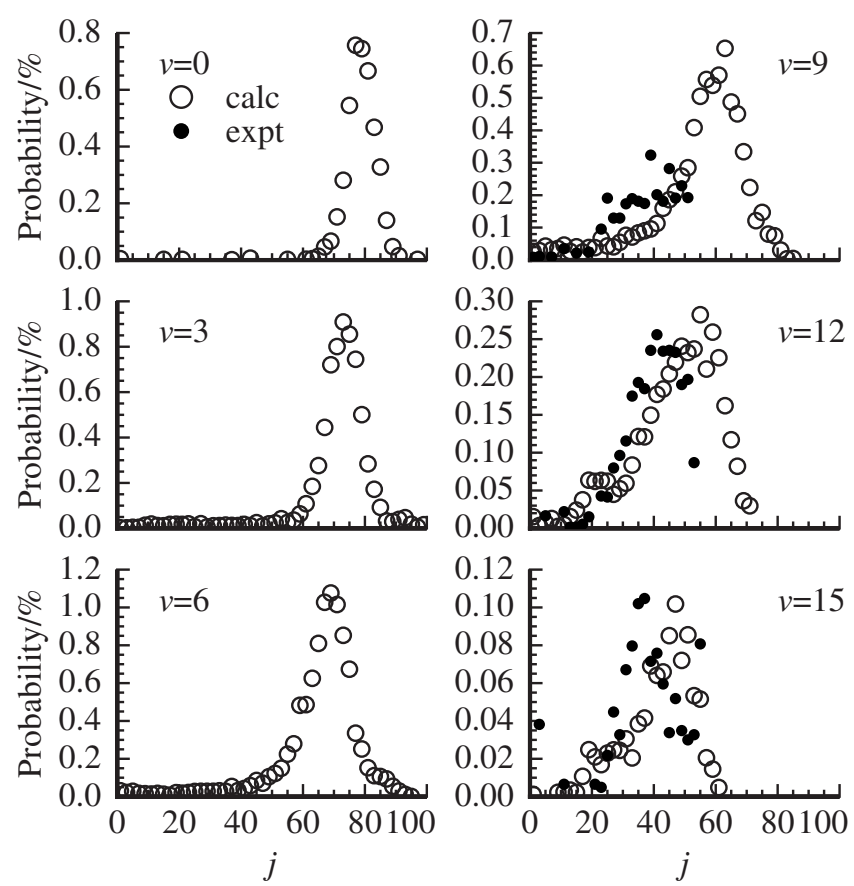

FIG. 8. Rotational distributions of $\mathrm{O}_{2}\left(X^{3} \Sigma_{g}^{-}\right)$the for $248 \mathrm{~nm}$ photodissociation. The ordinates specify the fraction of the total triplet state population represented by each $v / j$ state as extracted from the TSH calculation. The experimental data, from Fig. 10 of Daniels and Wiesenfeld (Ref. 28), have been independently scaled in each of the three right-hand panels to bring them onto the same scale as the computed distribution. pected for dissociation on a highly repulsive surface from a bent precursor. The TSH calculations agree with the experiment that the main maximum in the rotational distribution moves to a higher $j$ with decreasing $v$, and the widths of the main peaks agree reasonably well. (The experimental data for $v=9$ do not extend all the way to the maximum in the rotational distribution.) The computed maxima for $j=12$ and 15 appear at $j$ higher by roughly 10 quanta than the observed ones.

The computed rotational distributions at $v=12$ and 15 have small low- $j$ shoulders appearing near $j=20$. We believe these to be artifacts arising from the momentum adjustment needed for energy conservation at a surface hop. They are much stronger in our calculations using the earlier momentum adjustment method, ${ }^{12}$ and they disappear if we do not adjust the momenta at all but let the trajectory continue after the hop with a new total energy.

\section{SUMMARY}

We have applied the diabatic B and $\mathrm{R}$ state potential energy and coupling surfaces of Schinke and McBane ${ }^{12}$ and the fewest-switches TSH approach of Tully ${ }^{13}$ to the calculation of ozone UV photodissociation energy and angular distributions for the singlet products and energy distributions for the triplet products. Overall, the agreement with experimental measurements is very good, although some disagreements point out remaining weaknesses in the theory.

In the singlet channel, we have attributed the increase of the angular anisotropy parameter $\beta$ with wavelength observed by Dylewski et al. ${ }^{23}$ to the influence of the residual bending potential on the $\mathrm{B}$ state late in the photodissociation process. Since the computed $\beta$ are too high compared to the experiment, the residual bending potential is probably somewhat too strong; this suggestion is consistent with the computed singlet-channel rotational distributions, which peak at slightly too low $j$ values compared to experiment. The mechanism that produces the oscillatory rotational distributions in the singlet channel observed by Valentini et $a .^{25}$ is not included in the calculations.

In the triplet channel, the detailed calculations presented here confirm and extend the suggestion of Qu et al. ${ }^{11}$ that the dependence of the surface-hopping transition probability on the $\mathrm{O}_{2}$ bond length at the time the $\mathrm{B} / \mathrm{R}$ crossing seam is encountered has a dominant influence on the dynamics. That dependence can be understood accurately with a LandauZener treatment. The computed internal energy distributions of the triplet products agree fairly well with the measurements of Brouard et al. $;{ }^{26}$ in particular, the positions and widths of the main, low-energy parts of the distributions are reproduced accurately. However, the detailed structures of those distributions are sensitive to small changes in the B state surface, especially for long bond lengths, and are not captured accurately. In addition, the experimental distributions contain substantial intensity with $E_{\text {int }}>2.5 \mathrm{eV}$ that cannot be attributed to excitation to the Herzberg states ${ }^{27}$ and the origin of those product molecules is unknown. 
TABLE II. Components of the final position and velocity for each of the three atoms in space-fixed coordinates, defined in terms of quantities available from the trajectory calculations.

\begin{tabular}{cccc}
\hline \hline & Atom 1 & Atom 2 & Atom 3 \\
\hline$y$ & $y_{0}+f_{1} r \sin \beta$ & $y_{0}-\left(1-f_{1}\right) r \sin \beta$ & $-f_{3} R \sin \phi_{R}$ \\
$z$ & $z_{0}-f_{1} r \cos \beta$ & $z_{0}+\left(1-f_{1}\right) r \cos \beta$ & $-f_{3} R \cos \phi_{R}$ \\
$\dot{y}$ & $\dot{y}_{0}+f_{1}(\dot{r} \sin \beta+r \dot{\beta} \cos \beta)$ & $\dot{y}_{0}-\left(1-f_{1}\right)(\dot{r} \sin \beta+r \dot{\beta} \cos \beta)$ & $-f_{3}\left(\dot{R} \sin \phi_{R}+R \dot{\phi}_{R} \cos \phi_{R}\right)$ \\
$\dot{z}$ & $\dot{z}_{0}-f_{1}(\dot{r} \cos \beta-r \dot{\beta} \sin \beta)$ & $\dot{z}_{0}+\left(1-f_{1}\right)(\dot{r} \cos \beta-r \dot{\beta} \sin \beta)$ & $-f_{3}\left(\dot{R} \cos \phi_{R}-R \dot{\phi}_{R} \sin \phi_{R}\right)$ \\
\hline \hline
\end{tabular}

\section{ACKNOWLEDGMENTS}

G.C.M. and L.T.N. are grateful to the Michigan Space Grant Consortium and the Ott Scholars Fund for financial support. G.C.M. thanks M. Brouard and A. J. Johnsen for providing the data in Ref. 26 in tabular form, and G. E. Hall, P. L. Houston, and S. M. Dylewski for the discussions.

\section{APPENDIX: COORDINATE TRANSFORMATION}

To evaluate $\cos \theta_{\mathrm{m}}$ for a trajectory, we must compute the velocity vector of the outgoing atom in space-fixed coordinates. Our trajectories were carried out in internal Jacobi coordinates with one additional equation determining $\phi_{R}$, the final orientation angle of the $\boldsymbol{R}$ vector in a space-fixed frame. At the end of each trajectory, the Jacobi coordinates $r, R$, and $\gamma$, the angle $\phi_{R}$, and their time derivatives are available. The calculation of the final atomic velocities from those quantities is not difficult, but we can state from experience that it can be done clumsily. We have not seen the necessary expressions in literature, so we present them here.

We choose the space-fixed coordinate system so that its $z$ axis points along the initial $\boldsymbol{R}$ vector and its $y$ axis lies in the plane of the molecule (which, for $J=0$, is fixed in space throughout the trajectory). The origin of the space-fixed system lies at the center of mass of the three atoms. The $\boldsymbol{R}$ vector points from atom 3 toward the center of mass of atoms 1 and 2; $r$ is the distance between atoms 1 and 2. Figure 9 shows the relevant quantities.

We define the mass ratios $f_{1}=m_{2} /\left(m_{1}+m_{2}\right)$ and $f_{3}=\left(m_{1}\right.$ $\left.+m_{2}\right) /\left(m_{1}+m_{2}+m_{3}\right)$. (For ozone, $f_{1}=1 / 2$ and $f_{3}=2 / 3$.) Then, the center of mass of atoms 1 and 2 lies at $z_{0}$

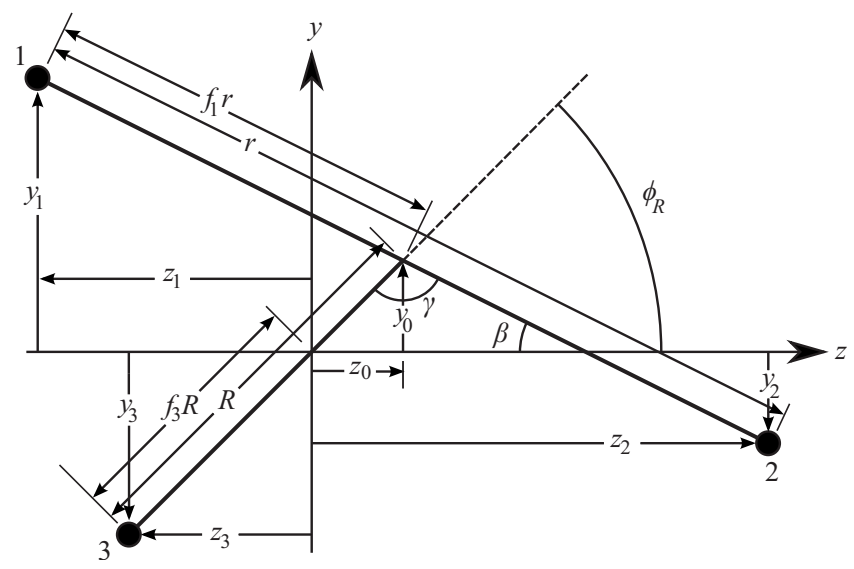

FIG. 9. Coordinate system for extraction of final atomic velocities. The $z$ axis points along the initial $\boldsymbol{R}$ vector; $\phi_{R}$ specifies the rotation of the $\boldsymbol{R}$ vector during the trajectory. $=\left(1-f_{3}\right) R \cos \phi_{R}$ and $y_{0}=\left(1-f_{3}\right) R \sin \phi_{R}$. The angle $\beta$ (not to be confused with the anisotropy parameter) is $\beta=\pi-\gamma$ $-\phi_{R}$. An inspection of Fig. 9 then yields the final $y$ and $z$ coordinates for each atom listed in Table II.

Chain rule differentiation with respect to time yields (indicating time derivatives with a dot) $\dot{\beta}=-\left(\dot{\gamma}+\dot{\phi}_{R}\right), \dot{y}_{0}$ $=\left(1-f_{3}\right)\left(\dot{R} \sin \phi_{R}+R \dot{\phi}_{R} \cos \phi_{R}\right)$, and $\dot{z}_{0}=\left(1-f_{3}\right)\left(\dot{R} \cos \phi_{R}\right.$ $\left.-R \dot{\phi}_{R} \sin \phi_{R}\right)$. The space-fixed velocity components $\dot{y}$ and $\dot{z}$ for each atom listed in the last two rows of Table II then follow by differentiation.

The transition dipole moment is a vector function of the initial molecular geometry. It may be tabulated in any of several different coordinate systems but it is generally straightforward to find its components $\mu_{z}$ and $\mu_{y}$ in the present coordinate system. Then, choosing the values of $\dot{y}$ and $\dot{z}$ associated with the atom that leaves, we have

$$
\cos \theta_{\mathrm{m}}=\frac{\boldsymbol{v} \cdot \boldsymbol{\mu}}{|\boldsymbol{v} \| \boldsymbol{\mu}|}=\frac{\dot{z} \mu_{z}+\dot{y} \mu_{y}}{\left(\left(\dot{y}^{2}+\dot{z}^{2}\right)\left(\mu_{y}^{2}+\mu_{z}^{2}\right)\right)^{1 / 2}} .
$$

Alternatively, one might compute orientation angles for the velocity $\phi_{v}=\tan ^{-1}(\dot{y} / \dot{z})$ and for the transition dipole moment $\phi_{\mu}=\tan ^{-1}\left(\mu_{y} / \mu_{z}\right)$ so that $\theta_{\mathrm{m}}=\phi_{v}-\phi_{\mu}$. Either of these procedures gives $\cos \theta_{\mathrm{m}}$ for a particular trajectory. The anisotropy parameter $\beta$ may then be calculated by averaging over trajectories, as described in Sec. II B.

${ }^{1}$ H. S. Johnston, Annu. Rev. Phys. Chem. 43, 1 (1992).

${ }^{2}$ Y. Matsumi and M. Kawasaki, Chem. Rev. (Washington, D.C.) 103, 4767 (2003).

${ }^{3}$ P. L. Houston, in Modern Trends in Chemical Reaction Dynamics: Experiment and Theory (Part 2), Advanced Series in Physical Chemistry Vol. 14, edited by X. Yang and K. Liu (World Scientific, Singapore, 2004).

${ }^{4}$ S. Y. Grebenshchikov, Z.-W. Qu, H. Zhu, and R. Schinke, Phys. Chem. Chem. Phys. 9, 2044 (2007).

${ }^{5}$ P. J. Hay, R. T. Pack, R. B. Walker, and E. J. Heller, J. Phys. Chem. 86, 862 (1982).

${ }^{6}$ M. G. Sheppard and R. B. Walker, J. Chem. Phys. 78, 7191 (1983).

${ }^{7}$ K. Yamashita, K. Morokuma, F. Le Quéré, and C. Leforestier, Chem. Phys. Lett. 191, 515 (1992).

${ }^{8}$ A. Banichevich, S. D. Peyerimhoff, and F. Grein, Chem. Phys. 178, 155 (1993).

${ }^{9}$ E. Baloïtcha and G. G. Balint-Kurti, J. Chem. Phys. 123, 014306 (2005); 128, 089901 (2008).

${ }^{10}$ Z. Qu, H. Zhu, S. Y. Grebenshchikov, and R. Schinke, J. Chem. Phys. 123, 074305 (2005).

${ }^{11}$ Z. Qu, H. Zhu, S. Y. Grebenshchikov, and R. Schinke, J. Chem. Phys. 122, 191102 (2005).

${ }^{12}$ R. Schinke and G. C. McBane, J. Chem. Phys. 132, 044305 (2010).

${ }^{13}$ J. C. Tully, J. Chem. Phys. 93, 1061 (1990).

${ }^{14}$ R. Schinke, Photodissociation Dynamics (Cambridge University Press, Cambridge, 1993).

${ }^{15}$ N. C. Blais and D. G. Truhlar, J. Chem. Phys. 79, 1334 (1983). 
${ }^{16}$ M. D. Hack, A. W. Jasper, Y. L. Volobuev, D. W. Schwenke, and D. G. Truhlar, J. Phys. Chem. A 103, 6309 (1999).

${ }^{17}$ J. C. Nieh and J. J. Valentini, J. Phys. Chem. 91, 1370 (1987).

${ }^{18}$ R. R. Laher and F. R. Gilmore, J. Phys. Chem. 20, 685 (1991).

${ }^{19}$ L. Bonnet and J.-C. Rayez, Chem. Phys. Lett. 277, 183 (1997).

${ }^{20}$ L. Bonnet and J.-C. Rayez, Chem. Phys. Lett. 397, 106 (2004).

${ }^{21}$ R. N. Zare, Angular Momentum: Understanding Spatial Aspects in Chemistry and Physics (Wiley, New York, 1988).

${ }^{22}$ A. V. Demyanenko, V. Dribinski, H. Reisler, H. Meyer, and C. X. W. Qian, J. Chem. Phys. 111, 7383 (1999).
${ }^{23}$ S. M. Dylewski, J. D. Geiser, and P. L. Houston, J. Chem. Phys. 115, 7460 (2001)

${ }^{24}$ S. Y. Lin, K. L. Han, and G. Z. He, J. Chem. Phys. 114, 10651 (2001).

${ }^{25}$ J. J. Valentini, D. P. Gerrity, D. L. Phillips, J. C. Nieh, and K. D. Tabor, J. Chem. Phys. 86, 6745 (1987).

${ }^{26}$ M. Brouard, A. Goman, S. J. Horrocks, A. J. Johnsen, F. Quadrini, and W.-H. Yuen, J. Chem. Phys. 127, 144304 (2007).

${ }^{27}$ R. Schinke, G. C. McBane, L. Shen, P. C. Singh, and A. G. Suits, J. Chem. Phys. 131, 011101 (2009).

${ }^{28}$ M. J. Daniels and J. R. Wiesenfeld, J. Chem. Phys. 98, 321 (1993). 